\title{
Autotaxin is a novel molecular identifier of type I endometrial cancer
}

\author{
Antonio Mazzocca' ${ }^{1}$ - Luca Maria Schönauer ${ }^{2} \cdot$ Rosalba De Nola $^{2,3} \cdot$ Antonio Lippolis $^{2} \cdot$ Teresa Marrano $^{2}$. \\ Matteo Loverro ${ }^{2} \cdot$ Carlo Sabbà $^{1} \cdot$ Edoardo Di Naro $^{2}$
}

Received: 10 September 2018 / Accepted: 25 October 2018 / Published online: 29 October 2018

(c) Springer Science+Business Media, LLC, part of Springer Nature 2018

\begin{abstract}
Endometrial cancer is the most common cancer of the female genital tract in Western Countries, with an incidence of 150.000 new cases/year. Despite high incidence, little is known about the molecular pathogenesis of this tumor. Phospholipids including lysophosphatidic acid (LPA) are involved in proliferation and dissemination of cancer. LPA is a potent bioactive phospholipid synthesized by autotaxin (ATX) through its lysophospholipase D activity. Recent evidence suggests that the ATX/LPA signaling axis plays a role in endometrial cancer. We carried out a prospective study involving two groups of patients classified in accordance to hysteroscopic-guided biopsy. Patients with histological diagnosis of endometrial cancer were enrolled into group one, whereas control patients with pelvic organ prolapse were assigned group two. Both groups underwent hysterectomy, with either open or laparoscopic surgery. After uterine extraction, a second endometrial biopsy was performed to collect tissues. Real-Time PCR was performed to evaluate ATX gene expression in collected tissues. Statistical analysis including unpaired two-way or one-way Student's $t$ test and ANOVA was performed. We found ATX gene expression significantly higher in neoplastic endometrium compared with normal tissue $(P$ value $=0.0002)$. In particular, the expression of ATX was significantly elevated in type I endometrial cancer (i.e., endometrioid histotype) compared to type II, in premenopausal women and in patients affected either by obesity (BMI > 30) or diabetes. We propose ATX as a novel potential biomarker particularly implicated in the pathobiology of type I endometrial cancer. Also, we propose ATX as a useful theranostic target in endometrial cancer.
\end{abstract}

Keywords Autotaxin (ATX) · Endometrial cancer · Lysophosphatidic acid (LPA) · Biological marker · Obesity

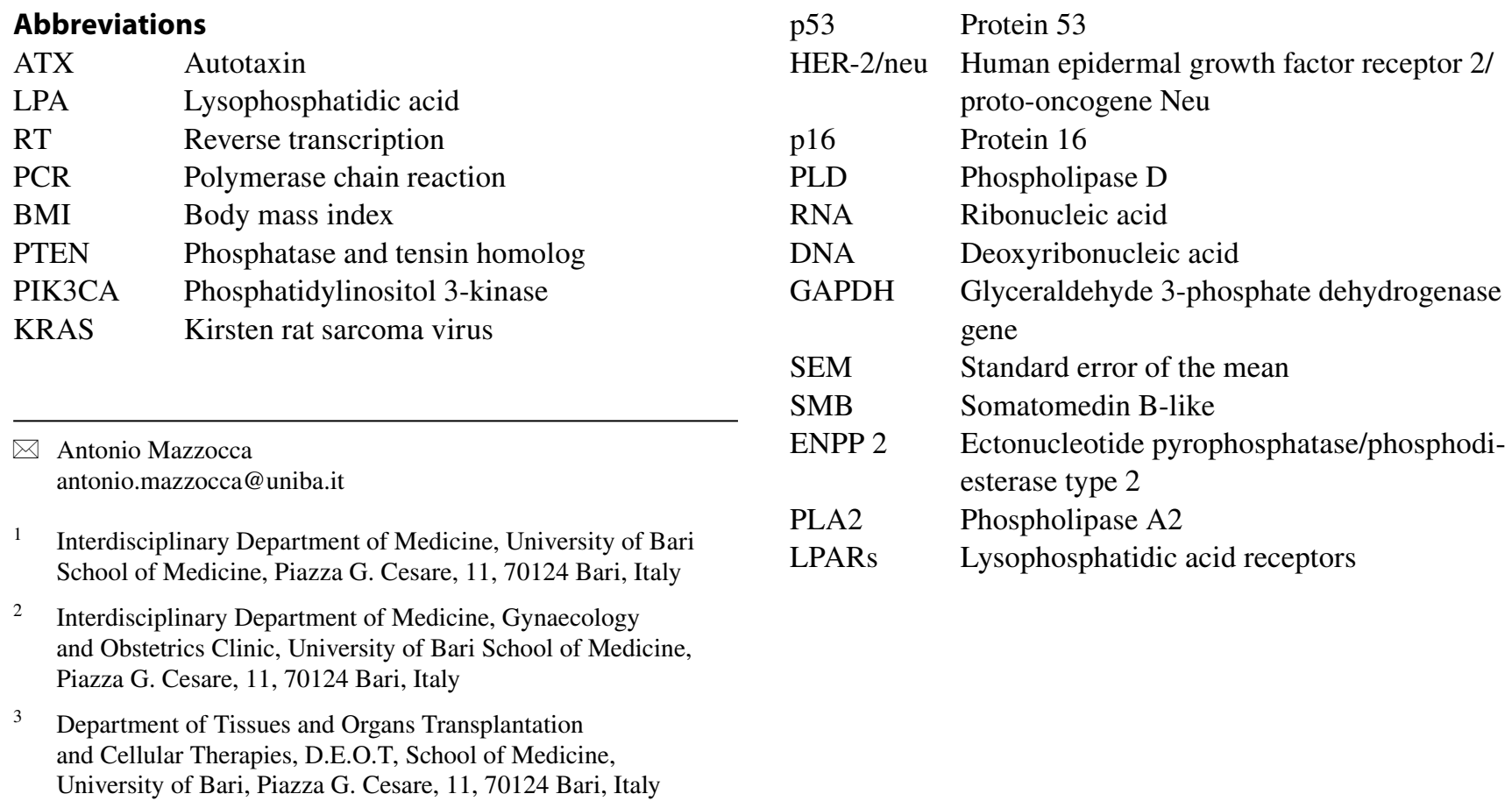




\section{Introduction}

Endometrial cancer is the most common neoplasm of the female genital tract in Western Countries, with a global incidence of 287,000 and a mortality rate of 74,000 per year in 2008 [1]. The endometrial cancer originates from the endometrial lining and glandular epithelium and occurs mainly at a mean age of 55-60 years. Nevertheless, epidemiological data have shown an increasing incidence of the disease both in under-50-year-old women $(1.5 \%)$ and in over-50s (2.6\%) from 2006 to 2010 , whereas the endometrial cancer mortality rate is increased of $1.5 \%$ among under 50s women and remained stable in over-50-year-old women [2]. This increasing incidence has been associated with an epidemic of obesity and absence of physical inactivity [3]. Two types of endometrial carcinoma have long been identified and they display differences in the field of pathology, biology, and clinics. In particular, type I is an estrogen-driven tumor affecting usually pre- and perimenopausal woman, characterized by endometrioid histological features, deriving from pre-malignant hyperplasia, with good prognosis (5-year survival $>90 \%$ ) and it harbors mutations in PTEN, PIK3CA, KRAS, b-catenin, and microsatellite instability. However, the type I incidence is facing a recent increase probably due to the pandemic obesity, which is a known risk factor for endometrial cancer. By contrast, type II represents only the $10 \%$ of all endometrial cancers and it is composed of non-endometrioid histotypes, namely in particular: papillary serous carcinomas, sarcomas, and clear cell carcinomas. The type II arises from an atrophic endometrium background and it occurs in an older onset age (about 10 years later than type I). Type II also has a worse prognosis (all stage have an overall survival of $35 \%$ ) and a trend of reduced survival in the last years, harboring genetic mutations in p53, HER-2/ neu, p16, and E-cadherin [4]. Moreover, all together endometrial cancer stages are characterized by a 5 -year overall survival of about $80 \%$, even without any valid screening method [3]. In Europe, one out 9000 women undergoes death for endometrial cancer, so it seems unlikely that this trend could decrease in the near future, probably due to the slight influence of early detection and kind of treatment on endometrial cancer mortality [3]. The main therapy for endometrial cancer is surgery followed, in selected cases, by radiotherapy to treat the microscopic disease of pelvic lymph nodes, the central pelvic region, and the upper vagina [3].

There is a growing interest among physicians in the standard radiotherapy, whose role has been re-evaluated in many studies (PORTEC-1, Post Operative Radiation Therapy in Endometrial Carcinoma Trial 1; GOG9, Gynaecologic Oncologic Group 9; ASTEC/EN.5, Adjuvant external beam radiotherapy in the treatment of endometrial cancer randomized trials). In advanced stages, adjuvant chemotherapy seems to be more effective than radiotherapy. Conversely, chemotherapy is associated with more side effects [5]. These data have encouraged the scientific community to focus on the physiopathology of endometrial cancer in order to find new therapeutic approaches. More recently, researchers are investigating the role of lipid metabolism in the pathogenesis of several solid cancers, including endometrial and ovarian cancer [6]. For example, dyslipidemia is frequently associated with conditions that promote endometrial cancer. Therefore, we focused on the role of phospholipids in this type of cancer [7]. Autotaxin (ATX), also known as ectonucleotide pyrophosphatase/phosphodiesterase family member 2 (ENPP 2), is a transmembrane phospholipase D (PLD) that catalyzes the hydrolysis of the distal phosphoester bond between monoacylglycerol phosphates and the polar head group of lysophospholipids, determining the biosynthesis of LPA. When the biosynthesis and the signaling pathway of LPA become altered, many features of cancer pathobiology, oncogenesis, and cancer progression can be affected [8]. In the present study, we investigated the ATX gene expression in the endometrium of normal and neoplastic perimenopausal women to clarify the role and the clinical significance of the ATX/LPA signaling pathway in this disease.

\section{Materials and methods}

\section{Patients}

This is a prospective study involving 55 patients, subdivided into two groups (normal and neoplastic endometrium) who came to our attention during a 6-month period starting from January to July 2015 , treated with total hysterectomy, for uterine prolapse without any endometrial pathology o previous endometrial cancer diagnosis. Patients were divided into two groups based on histopathology: (i) the first group included 26 patients with diagnosis of endometrial cancer; (ii) the second group of 29 patients with a normal endometrium. Ultrasound and MRI evaluation were performed as pre-operative assessment in case of endometrial cancer. Endometrial cancer was treated with type A hysterectomy, bilateral salpingooophorectomy, and pelvic/lomboaortic lymphadenectomy in medium or high-risk patients [9]. Patients with prolapse underwent simple hysterectomy with or without adjunctive surgical procedure to restore normal pelvic support. Exclusion criteria encompass previous or coexistent tumors and concomitant suspicious adnexal masses. We collected data about clinical history, previous pregnancies, age, and BMI. 
Each patient underwent clinical and laboratory investigations including trans-vaginal ultrasound examination; hysteroscopy with endometrial biopsy; Pap test, and serum oncological markers. Informed consent was given by all patients enrolled in the study. Endometrial biopsy was performed for each uterine extraction and the collected sample tissues were stored at $-80{ }^{\circ} \mathrm{C}$. Endometrial cancers were classified into different stages and histological subtype (Table 1) according to the International Federation of Gynecology and Obstetrics Consensus of 2009 [10]. Patients with endometrial cancer were subjected to a clinical follow-up every 3-6 months for at least 30 months. The ATX expression levels were evaluated in both the groups: patients with endometrial cancer and women with normal endometrium. The level of ATX expression in endometrial cancer samples was then correlated with histological type, stage of the disease, BMI, menopause onset age, type II diabetes, and hypertension.

Table 1 Clinico-pathological characteristics of patients with endometrial cancer enrolled in the study

\begin{tabular}{lc}
\hline Population under study: features & No. \\
\hline Pre-operative diagnosis & 26 \\
Endometrial cancer (EC) & 29 \\
Benign conditions (genital prolapse) & \\
Hormonal status in endometrial cancer group & 23 \\
Post-menopause & 3 \\
Pre-menopause & 12 \\
Endometrioid adenocarcinoma of the endometrium & 2 \\
IA G1 & 1 \\
IA G2 & 3 \\
IA G3 & 4 \\
IB G1 & \\
II G2-II G3-III C & 1 \\
Special histotypes of the endometrial cancer & 3 \\
Clear cell carcinoma & \\
Sarcoma & 16 \\
BMI in the endometrial cancer group & 15 \\
BMI < 30 & 11 \\
BMI > 30 & \\
Onset of menopause in the endometrial cancer group & \\
Before 55 years (regular onset) & \\
After 55 years (late onset) & \\
Diabetes in the EC group & \\
Diabetic women & \\
Non-diabetic women & \\
Blood pressure in the EC group & \\
Normal blood pressure & \\
Hypertension & \\
\hline & \\
\hline
\end{tabular}

\section{Sample collection and RNA extraction}

Frozen tissues were homogenized in TriReagent ${ }^{\circledR}$ (Molecular Research Center) and RNA was precipitated with isopropanol, applied to RNeasy spin columns (Qiagen), eluted, and treated with RNase-free DNase for $30 \mathrm{~min}$ at $37 \mathrm{C}$, followed by heat inactivation at $75^{\circ} \mathrm{C}$ and storage at $-80^{\circ} \mathrm{C}$.

\section{Quantitative real-time PCR}

Total RNA was isolated using the RNeasy Mini Kit (Qiagen) and underwent DNAse treatment (TURBO DNA-free ${ }^{\text {TM }}$ Ambion $^{\circledR}$ ). Reverse Transcription (RT) was then carried out with random primers using $1 \mu \mathrm{g}$ of total RNA from each sample. Quantitative RT-PCR was performed using predesigned primers and SYBR Green ${ }^{\circledR}$ master mix (Biorad). Housekeeping Glyceraldehyde 3-phosphate dehydrogenase gene (GAPDH) was used as endogenous control. ATX/PLD Primers were obtained from Integrated DNA Technologies ${ }^{\circledR}$ (Leuven, Belgium): Forward: 5'-TGA CGG CCC TCT CTC TGT GT-3'. Reverse: 5'-ATT GCA GCT CTC CTC GTT GTC-3'.

\section{Statistical analyses}

For correlations, Pearson's $r$ coefficients were calculated. Data are represented as the mean \pm SEM. Significant differences between groups were determined by unpaired twoway or one-way Student's $t$ test or ANOVA. $P<0.05$ was considered statistically significant. The study was approved by local Independent Ethics Committee of the Polyclinic Consortium University Hospital, University of Bari. The patients gave prior written informed consent to the use of their tissues for clinical research.

\section{Results}

To evaluate the involvement of ATX in endometrial cancer, we carried out analysis in endometrial tissues by using quantitative PCR. We found that ATX expression levels were significantly higher in endometrial cancer compared with control samples $(P$ value $=0.0002)$ as shown in Fig. 1a. Surprisingly, more elevated values of ATX were observed in patients with endometrioid histotype compared with special histotypes ( $P$ value $=0,038$ ) (Fig. 1b). Furthermore, we found a higher rate of ATX expression in premenopausal population, compared to post-menopausal women with endometrial cancer ( $P$ value of 0.0437 ) (Fig. 1c). Metabolic diseases are often associated to endometrial cancer. To assess the role of ATX in endometrial cancer patients with these metabolic conditions, we performed quantitative PCR in RNA extracted from endometrial biopsies. The 
A

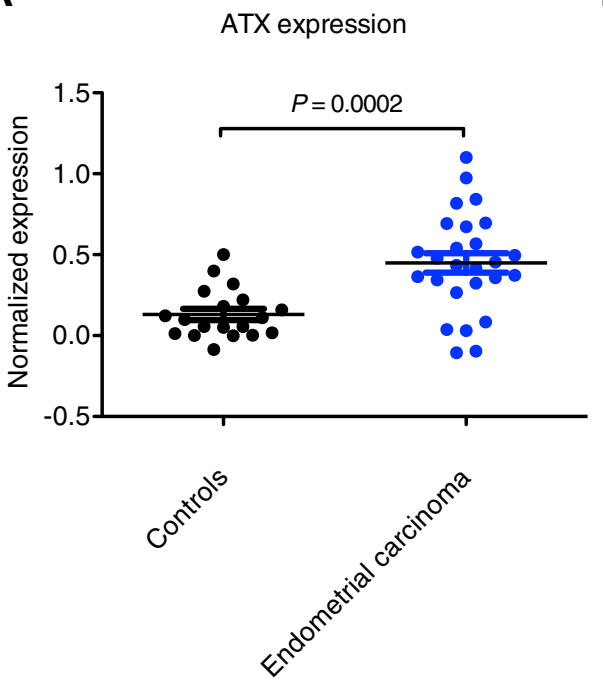

B

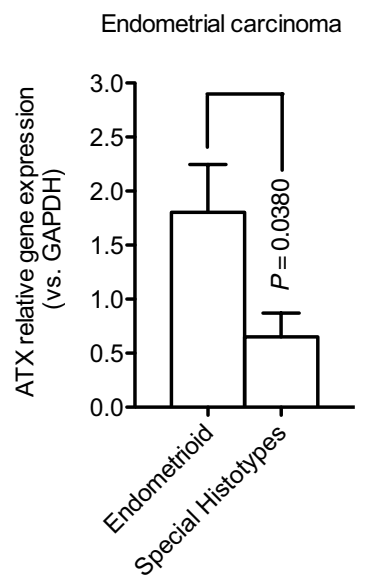

C

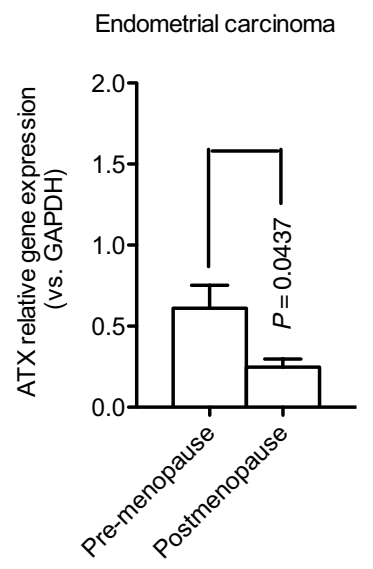

Fig. 1 ATX expression levels evaluated by a real-time PCR analysis in patients with endometrial cancer. a ATX expression levels were significantly higher in endometrial cancer tissues compared with controls $(P$ value $=0.0002)$. $\mathbf{b}$ Increase of ATX expression in endometri- oid histotype compared to special histotypes $(P$ value $=0.038)$. $\mathbf{c}$ ATX expression levels were higher in women who had received the diagnosis of endometrial cancer before menopause compared with postmenopause patients diagnosed with endometrial cancer

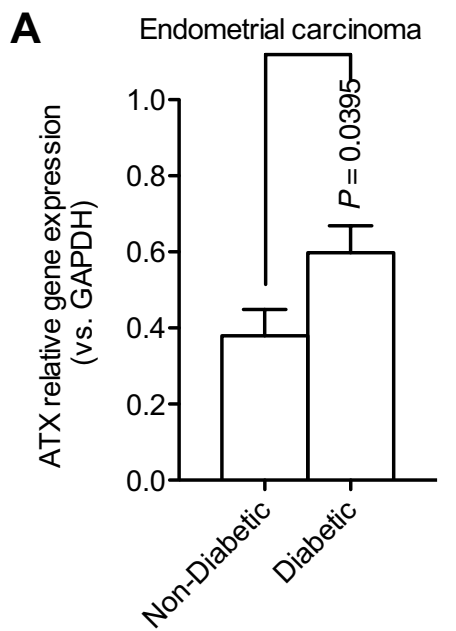

Fig. 2 ATX expression in metabolic conditions associated with endometrial cancer. a Significant increase of ATX expression in diabetic patients with endometrial cancer $(P$ value $=0.0395)$. $\mathbf{b}$ Obese patients with endometrial cancer show a significant higher expression of

comparative analysis showed elevated ATX expression in diabetic $(P$ value $=0.0395)$ compared with non-diabetic patients (Fig. 2a). In addition, we found a highly significant increased in ATX expression $(P$ value $=0.0021)$ when we compare obese versus non-obese patients with endometrial cancer (Fig. 2b). A further analysis revealed that ATX expression levels were significantly higher in obese

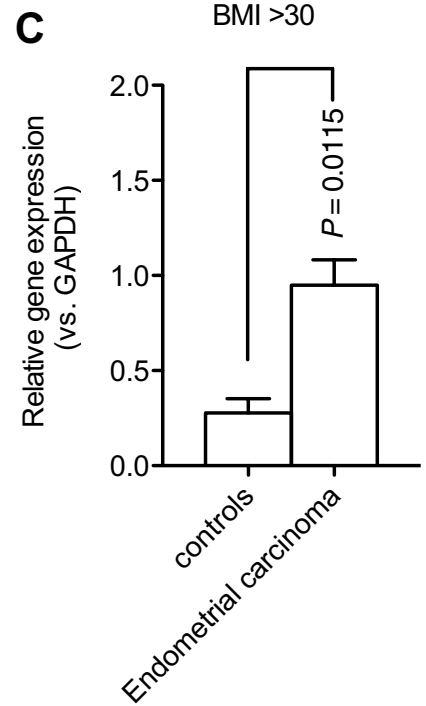

endometrial ATX $(P$ value $=0.0115)$. $\mathbf{c}$ Significant higher expression of endometrial ATX was observed in obese patients compared to nonobese with endometrial carcinoma $(P$ value $=0.0021)$

women $(\mathrm{BMI} \geq 30)$ with endometrial cancer compared with obese women with normal endometrium $(P$ value $=0.0115)$ (Fig. 2c). Next, we evaluated the correlation of ATX expression with cancer stage. The expression levels of ATX did not show any significant difference across various stages of endometrial cancer. However, although non-significant, a trend showing increased ATX expression levels was 

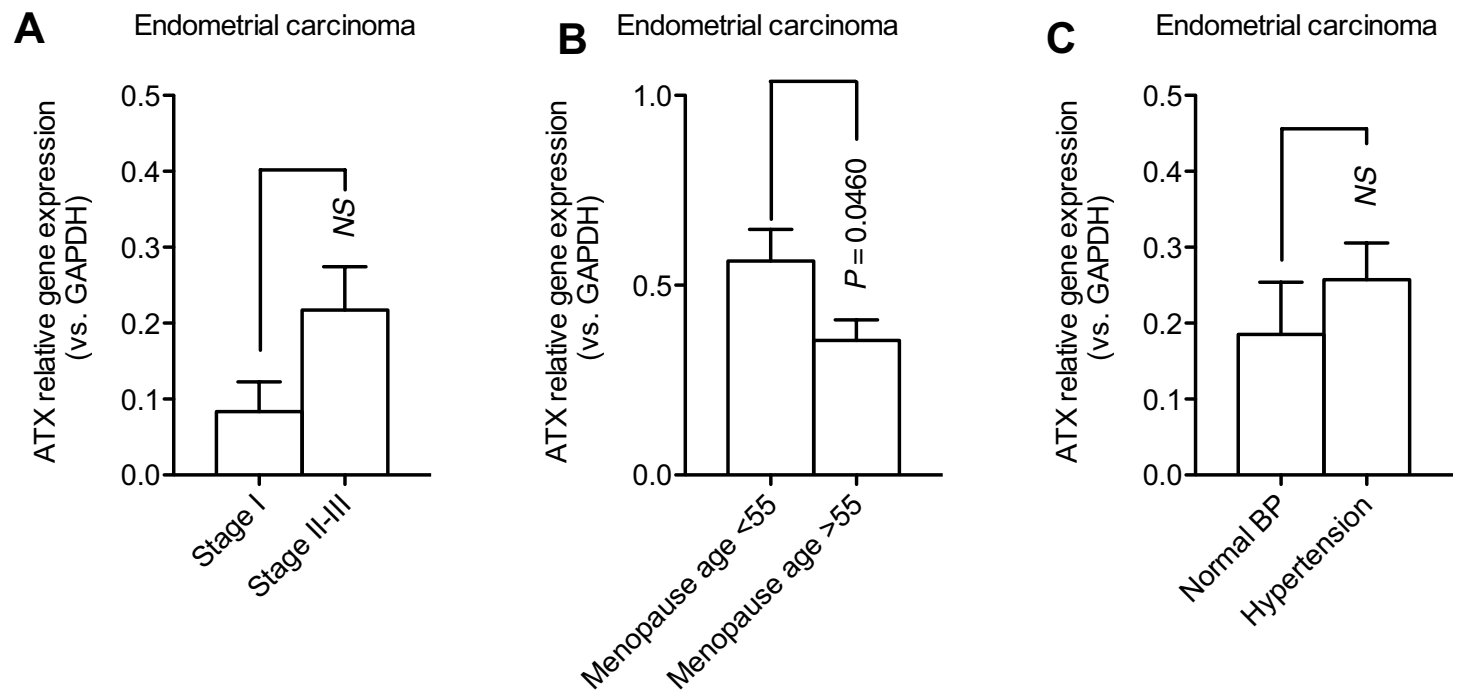

Fig. 3 ATX expression evaluated by real-time PCR analysis in different subgroups of endometrial cancer. a Although non-significant, an increased trend in ATX expression was observed in advanced stages (II-III) compared with early stages (I) of endometrial cancer. b Significant $(P=0.0460)$ higher ATX expression in endometrial cancer diagnosed in women with normal menopausal onset (before 55 years), compared to endometrial cancer diagnosed in women with late menopausal onset (post 55 years). c Absence of ATX significant endometrial expression in women with or without hypertension observed in advanced (II-III) compared to early stages (I) (Fig. 3a). Lastly, we found a significant ATX expression $(P=0.0460)$ in patients with endometrial cancer with normal menopausal onset (before 55 years) compared to those with late menopausal onset (post 55 years) (Fig. 3b). Lack of statistical significance was found in ATX results, when we compared patients with endometrial cancer with or without hypertension (Fig. 3c).

\section{Discussion}

In the present study, our main goal was to investigate the role of ATX expression in normal and neoplastic endometrium. We found a significant difference between these two groups with elevated ATX expression in endometrial cancer. We have also shown that ATX is significantly increased in patients with endometrial cancer associated to hormonal status and metabolic conditions including obesity and diabetes.

ATX, originally discovered as an autocrine factor promoting chemotaxis in melanoma cells, is a $100 \mathrm{kDa}$ multi-domain glycoprotein, consisting of two N-terminal somatomedin B-like (SMB) domains, a central catalytic phosphodiesterase domain and a C-terminal nuclease-like domain, which acts as an ecto-lysophospholipase D. ATX is encoded by the ENPP2 gene, which occupies a 116 kilobase-long DNA segment of human chromosome 8 [11-14]. The product of the ATX catalysis is LPA, a small glycerophospholipid (1-acyl-2-hydroxy-sn-glycero-3-phosphate) generated from the cleavage of the choline/serine head group from lysophosphatidylcholine or from lysophosphatidylserine [15]. Although LPA can be produced by different enzymes (i.e., PLA2, phospholipase A2), the main source of all circulating bioactive LPA is due to the enzymatic activity of ATX [8]. LPA acts on different LPAspecific $\mathrm{G}$ protein-coupled receptors eliciting a great variety of cellular responses $[16,13]$.

LPA and ATX have been also studied in the pathogenesis of cancer, since they are involved in various processes essential for cancer progression, such as migration, invasion, tumor cell survival, and angiogenesis [17-19]. The ATX/LPA signaling axis promotes the proliferation and the dissemination of ovarian cancer and evidence from the present study and other studies indicate that it could be involved in the pathobiology of endometrial cancer [20]. In the gynecological field, LPA is thought to be a tumorpromoting factor, inducing, for example, the proliferation and the mitotic activity of leiomyomas [21, 22]. Indeed, ATX influences the functions and the proliferation of the muscular uterine components because of the synthesis of LPA in the smooth muscle cells [22]. Interestingly, ATX could interact with the cellular microenvironment inducing many biological effects. ATX also binds to integrins through the SMB-like domains and modulates the mitogenic activity of cancer in mouse models [8]. Seo et al. revealed that ATX is highly secreted by ovarian cancer stem cells and that either drug inhibition or ATX gene suppression reduces the LPA biosynthesis and consequently 
the ability of self-renewing in ovarian cancer stem cells and chemoresistance [23].

In the present study, we show that ATX RNA is more expressed in endometrial cancer compared with controls (pelvic organ prolapses). A previous study conducted by Wasniewski demonstrated a positive correlation $(P<0.005)$ of endometrial ATX/LPA signaling axis with endometrial cancers [20]. Wasniewski et al. carried out an interesting study based on the comparison of mRNA and protein levels of LPARs (lysophosphatidic acid receptors), ATX and PLA2, between type I endometrial cancers and normal endometrium samples (control), using qRT-PCR and western blotting. The first group was made of 37 post-menopausal women who underwent total abdominal hysterectomy because of endometrial cancers, whereas the second group (controls) was composed of 10 premenopausal women who underwent hysterectomy for leiomyomas. Regarding the various endometrial histotypes, we found that the expression of ATX gene is significantly elevated in the endometrioid endometrial cancer when compared to "special" histotypes. This clear difference reflects the pathogenic and the clinical divergence between the two histotypes, related to distinct metabolic and endocrine milieu. Endometrial cancers have long been classified into estrogen-dependent type I and estrogen-independent type II. The Type II is less common but clinically more aggressive than Type I, affecting older women, usually without any metabolic derangement or preexisting pre-cancerous lesions (hyperplasia with atypia), harboring p53 mutations, but with a wild PTEN phenotype [3, 24, 25]. Our data corroborate the aforementioned observations, in fact in patients with endometrial cancer, we found a remarkable elevated ATX gene expression in pre-menopause women still exposed to estrogenic milieu compared with those with a post-menopausal status, thus confirming the negative effect of estrogens in a dysmetabolic condition. This suggests a long developmental time for endometrioid endometrial cancer, whose roots mainly lie in the estrogen exposure during premenopausal period among genetically and metabolically predisposed women. In premenopausal women, overweight determines insulin resistance, excessive ovarian androgen biosynthesis, anovulation, and chronic progesterone deficiency [3]. Unlike Wasniewki et al. that enrolled only post-menopausal cases of endometrial cancer, we had the possibility to evaluate endometrial cancer in both pre-menopause and post-menopause status. Therefore, we correlated the hormonal influence in the pathogenesis of endometrial cancer and the higher ATX expression found in premenopausal patients. Moreover, we confirmed the correlation between type I endometrial cancer and ATX expression.

In our study, we also evaluated the role of metabolic diseases in endometrial cancer, in particular type II diabetes and obesity. We demonstrated a significantly higher expression of ATX in diabetic patients. This is in contrast with findings by Ref. [20]. The absence of correlation between diabetes and ATX expression in the study by Wasniewski may be due to the cohort that was mainly composed of over-60-yearold obese women with other conditions related to aging and dysmetabolism. Our observations are instead in line with the well-known correlation between ATX, insulin resistance, and metabolic syndrome [26]. Among women with diabetes, LPA downregulates blood glucose levels by triggering translocation of Glucose transporter type 4 to the plasma membrane of myocytes and adipocytes. This determines an increased dose-dependent glucose uptake in muscles and adipose tissues [27]. In dysmetabolic mice, this may apparently suggest a possible advantage. However, it can eventually cause deleterious effect by inhibiting glucoseinduced insulin secretion. Indeed, a chronic pharmacological inhibition with Ki16425 of LPA receptors improved glucose homeostasis in obese/pre-diabetic mice [19]. Nevertheless, in the present study, we do not show laboratory information supporting the potential correlation with insulin resistance in such patients. Due to the known correlation between high values of BMI and endometrial endometrioid cancer [10, 26], we decided to study the expression of the endometrial ATX gene expression in obese women, by comparing controls (healthy endometrium) with patients with endometrial cancer. Remarkably, ATX gene expression was higher in obese endometrial cancer group compared with controls similarly to Wasniewski et al. [20], who demonstrated an up-regulation of all the investigated LPARs (1-4) and of the LPA biosynthetic enzymes (ATX, PLA2) in endometrial cancer tissues. Next, we evaluated the entire group of endometrial cancer divided into two subgroups, endometrial cancer patients with $\mathrm{BMI}<30$ versus endometrial cancer patients with $\mathrm{BMI} \geq 30$, and we confirmed the statistically significant positive correlation between obesity and ATX gene expression. Undoubtedly, obesity induces a hyperestrogenic status with a continuous peripheral oestrone production in the adipose tissue associated with an imbalanced secretion of pro-inflammatory cytokines that have a supposed triggering role in the pathogenesis of endometrial cancer [26]. Interestingly, our study also shows a significantly higher value of ATX expression in endometrial cancer diagnosed in women with normal menopausal onset (before 55 years), compared to endometrial cancer diagnosed in women with late menopausal onset (post 55 years). This could be due to the longer exposure of endometrium to dysregulated metabolic and imbalanced endocrine status correlated to the late onset of menopause in the former group, known as a promoting factor of type I endometrial cancer $[28,29,26]$. All these factors confirm that endometrioid endometrial cancer is an estrogen correlated disease with a long latent phase characterized by dysmetabolic conditions of hyperinsulinemia (related to metabolic syndrome), 
type II diabetes and obesity. This dysmetabolic conditions is also characterized by a prolonged estrogen production within the adipose tissue among perimenopausal period in genetically predisposed subjects. Furthermore, obesity promotes an imbalanced level of insulin-like growth factor and its binding proteins. This condition can be enhanced mainly by ovarian oestradiol in pre-menopause or mostly by a peripheral transformation of oestron within the adipose tissue in post-menopause. In addition, this vicious cycle can be sustained by an insufficient biosynthesis of progesterone. Notably, the transcriptional activity of estrogen-receptors can be induced by insulin-like growth factor 1 even without sustained levels of oestradiol (i.e., in post-menopause), supporting the evidence of a cross-talk between these different pathways [3]. Finally, we suppose that ATX expression could act as a metabolic link between estrogen milieu and insulin resistance, mainly in obese women acting as a promoting factor in endometrial carcinogenesis. Despite its apparent low aggressiveness, endometrial cancer is often related to a considerable and persistent mortality. For this reason, researchers are trying to elucidate the causes that determine mortality of this form of gynecological cancer. Therefore, new insights into pathophysiology of endometrial cancer are urgently needed to identify new targets for more effective therapeutic interventions than those available today. These considerations led us to study the role of ATX expression in endometrial cancer. Notably, we found an increased expression of ATX gene in endometrial cancer, particularly in patients with endometrioid histotypes, BMI $\geq 30$, type II diabetes, pre-menopause, and regular menopause. Given the high 5-year survival rate (approximately about 95\%) [3] of early-stage endometrial cancer, we hypothesize that ATX expression, evaluated on endometrial biopsies or serum, as already demonstrated for LPA in ovarian cancer [17] may become an early marker for endometrial cancer detection. Similarly, a targeted therapy directed against ATX could be postulated as an effective tool in the treatment of endometrioid carcinoma, especially in the early stages.

Acknowledgements The work was supported by AIRC (Italian Association for Cancer Research) Investigator Grant (IG) 2015 Id.17758 (to A. Mazzocca).

Author contributions AM conceived and designed the study, planned and performed experiments, analyzed data, and wrote the manuscript. RDN, TM, analyzed data, reviewed the literature, and wrote the manuscript. AL collected all the endometrial samples and organized data. ML and CS helped with discussions and comments. LMS and EDN performed ultrasound, clinical evaluation, hysteroscopic biopsies, and the surgeries.

\section{Compliance with ethical standards}

Conflict of interest The authors declare no conflicts of interest.

\section{References}

1. Ferlay J, Shin HR, Bray F, Forman D, Mathers C, Parkin DM. Estimates of worldwide burden of cancer in 2008: GLOBOCAN 2008. Int J Cancer. 2010;127(12):2893-917.

2. Raspagliesi F, Gadducci A, Franchi M. Requisiti minimi per il trattamento delle neoplasie ginecologiche; 2007.

3. Amant F, Moerman P, Neven P, Timmerman D, Van Limbergen E, Vergote I. Endometrial cancer. The Lancet. 2005;366(9484):491-505.

4. Evans T, Sany O, Pearmain P, Ganesan R, Blann A, Sundar S. Differential trends in the rising incidence of endometrial cancer by type: data from a UK population-based registry from 1994 to 2006. Br J Cancer. 2011;104(9):1505.

5. Galaal K, Al MM, Bryant A, Lopes AD, Lawrie TA. Adjuvant chemotherapy for advanced endometrial cancer. Cochrane Database Syst Rev. 2014;5:CD010681.

6. Sonoda K. Molecular biology of gynecological cancer. Oncol Lett. 2016;11(1):16-22.

7. Fujita T, Miyamoto S, Onoyama I, Sonoda K, Mekada E, Nakano H. Expression of lysophosphatidic acid receptors and vascular endothelial growth factor mediating lysophosphatidic acid in the development of human ovarian cancer. Cancer Lett. 2003;192(2):161-9.

8. Federico L, Jeong KJ, Vellano CP, Mills GB. Thematic review series: phospholipases: central role in lipid signaling and disease: autotaxin, a lysophospholipase D with pleomorphic effects in oncogenesis and cancer progression. J Lipid Res. 2016;57(1):25.

9. Querleu D, Morrow CP. Classification of radical hysterectomy. Lancet Oncol. 2008;9(3):297-303.

10. Colombo N, Creutzberg C, Amant F, Bosse T, González-Martín A, Ledermann J et al. ESMO-ESGO-ESTRO consensus conference on endometrial cancer: diagnosis, treatment and follow-up. Ann Oncol. 2015;27(1):16-41.

11. Aoki J. Mechanisms of lysophosphatidic acid production. In Seminars in cell and developmental biology, vol. 15, no. 5. Cambridge: Academic Press; 2004, pp. 477-89.

12. Bächner D, Ahrens M, Betat N, Schröder D, Gross G. Developmental expression analysis of murine autotaxin (ATX). Mech Dev. 1999;84(1-2):121-5.

13. Hausmann J, Christodoulou E, Kasiem M, De Marco V, Van Meeteren LA, Moolenaar WH, et al. Mammalian cell expression, purification, crystallization and microcrystal data collection of autotaxin/ENPP2, a secreted mammalian glycoprotein. Acta Crystallogr Sect F. 2010;66(9):1130-5.

14. Okudaira S, Yukiura H, Aoki J. Biological roles of lysophosphatidic acid signaling through its production by autotaxin. Biochimie. 2010;92(6):698-706.

15. Gotoh M, Fujiwara Y, Yue J, Liu J, Lee S, Fells J, et al. Controlling cancer through the autotaxin-lysophosphatidic acid receptor axis; 2012.

16. Aoki J, Inoue A, Okudaira S. Two pathways for lysophosphatidic acid production. Biochim Biophy Acta. 2008;1781(9):513-8.

17. Choi JW, Herr DR, Noguchi K, Yung YC, Lee CW, Mutoh T et al. LPA receptors: subtypes and biological actions. Annu Rev Pharmacol Toxicol. 2010;50:157-86.

18. Li TT, Alemayehu M, Aziziyeh AI, Pape C, Pampillo M, Postovit LM, et al. $\beta$-Arrestin/Ral signaling regulates lysophosphatidic acid-mediated migration and invasion of human breast tumor cells. Mol Cancer Res. 2009;7(7):1064-77.

19. Rancoule C, Attane C, Gres S, Fournel A, Dusaulcy R, Bertrand $\mathrm{C}$, et al. Lysophosphatidic acid impairs glucose homeostasis and inhibits insulin secretion in high-fat diet obese mice. Diabetologia. 2013;56(6):1394-402. 
20. Wasniewski T, Woclawek-Potocka I, Boruszewska D, KowalczykZieba I, Sinderewicz E, Grycmacher K. The significance of the altered expression of lysophosphatidic acid receptors, autotaxin and phospholipase A2 as the potential biomarkers in type 1 endometrial cancer biology. Oncol Rep. 2015;34(5):2760-7.

21. Billon-Denis E, Tanfin Z, Robin P. Role of lysophosphatidic acid in the regulation of uterine leiomyoma cell proliferation by phospholipase D and autotaxin. J Lipid Res. 2008;49(2):295-307.

22. Tokumura A, Majima E, Kariya Y, Tominaga K, Kogure K, Yasuda K, Fukuzawa K. Identification of human plasma lysophospholipase D, a lysophosphatidic acid-producing enzyme, as autotaxin, a multifunctional phosphodiesterase. J Biol Chem. 2002;277(42):39436-42.

23. Seo EJ, Kwon YW, Jang IH, Kim DK, Lee SI, Choi EJ, et al. Autotaxin regulates maintenance of ovarian cancer stem cells through lysophosphatidic acid-mediated autocrine mechanism. Stem Cells. 2016;34(3):551-64.

24. Binder PS, Mutch DG. Update on prognostic markers for endometrial cancer. Women's Health. 2014;10(3):277-88.
25. Di Saia PJ, Creasman WT, Mannel RS, McMeekin DS, Mutch DG. Clinical gynecologic oncology e-book. Amsterdam: Elsevier Health Sciences; 2017

26. Setiawan VW, Yang HP, Pike MC, McCann SE, Yu H, Xiang YB, et al. Type I and II endometrial cancers: have they different risk factors? J Clin Oncol. 2013;31(20):2607.

27. Yea K, Kim J, Lim S, Park HS, Park KS, Suh PG, Ryu SH. Lysophosphatidic acid regulates blood glucose by stimulating myotube and adipocyte glucose uptake. J Mol Med. 2008;86(2):211-20.

28. D'Amore S, Palasciano G, Moschetta A. Peroxisome proliferatoractivated receptors (PPARS), metabolic sindrom and atherosclerosis. G Ital Arterioscler. 2013;4(2):8-22.

29. Reeves VL, Trybula JS, Wills RC, Goodpaster BH, Dubé JJ, Kienesberger PC, Kershaw EE. Serum autotaxin/ENPP 2 correlates with insulin resistance in older humans with obesity. Obesity. 2015;23(12):2371-6. 